\title{
Structure of the Space of Diametrically Complete Sets in a Minkowski Space
}

\author{
José Pedro Moreno • Rolf Schneider
}

Received: 6 July 2011 / Revised: 7 November 2011 / Accepted: 13 December 2011 /

Published online: 15 February 2012

(C) Springer Science+Business Media, LLC 2012

\begin{abstract}
We study the structure of the space of diametrically complete sets in a finite dimensional normed space. In contrast to the Euclidean case, this space is in general not convex. We show that its starshapedness is equivalent to the completeness of the parallel bodies of complete sets, a property studied in Moreno and Schneider (Isr. J. Math. 2012, doi:10.1007/s11856-012-0003-6), which is generically not satisfied. The space in question is, however, always contractible. Our main result states that in the case of a polyhedral norm, the space of translation classes of diametrically complete sets of given diameter is a finite polytopal complex. The proof makes use of a diagram technique, due to $\mathrm{P}$. McMullen, for the representation of translation classes of polytopes with given normal vectors. The paper concludes with a study of the extreme diametrically complete sets.
\end{abstract}

Keywords Minkowski space - Diametrically complete set - Set of constant width · Polyhedral norm $\cdot$ Polytopal complex $\cdot$ Extreme diametrically complete set

\section{Introduction}

A compact convex set in a finite dimensional real normed vector space (briefly, a Minkowski space) is said be of constant width if any two parallel supporting hyperplanes of the set have the same distance. A bounded set is called diametrically complete (briefly, complete) if it is not properly contained in a set of the same diameter. Clearly, a complete set is convex and compact. In Euclidean space, the two notions,

J.P. Moreno ( $\varangle)$

Dpto. Matemáticas, Facultad de Ciencias, Universidad Autónoma de Madrid, 28049 Madrid, Spain e-mail: josepedro.moreno@uam.es

R. Schneider

Mathematisches Institut, Albert-Ludwigs-Universität, 79104 Freiburg im Breisgau, Germany

e-mail: rolf.schneider@math.uni-freiburg.de 
constant width and diametrically complete, are equivalent, and bodies of constant width have a long and rich history. We refer the reader to the survey articles [2,6]. The survey by Martini and Swanepoel [9] contains a longer section on bodies of constant width in Minkowski spaces. In an arbitrary Minkowski space, every convex body of constant width is complete, but not conversely (except in dimension two); this was first shown by Eggleston [3]. It is not difficult to find Minkowski spaces where the only bodies of constant width are the balls of the space, whereas in every Minkowski space there are so many complete sets that every bounded set is contained in a complete set of the same diameter (not a ball, in general). For this reason, in general Minkowski spaces the study of complete sets should replace the study of bodies of constant width in Euclidean space. In the following, we initiate a study of the space of all complete sets in a Minkowski space. It is considerably more intricate than the space of bodies of constant width in a Euclidean space.

An $n$-dimensional Minkowski space (we assume $n \geq 2$ ) can be represented as $X=$ $\left(\mathbb{R}^{n},\|\cdot\|\right)$ with a norm $\|\cdot\|$. The set $B:=\left\{x \in \mathbb{R}^{n}:\|x\| \leq 1\right\}$ is the unit ball of $X$, and all sets $\lambda B+t$ with $\lambda>0$ and $t \in \mathbb{R}^{n}$ are called balls.

By $\mathcal{K}^{n}$ we denote the set of convex bodies (nonempty, compact, convex subsets) of $\mathbb{R}^{n}$, equipped with the topology of the Hausdorff metric induced by the given norm (or any other norm-this yields the same topology). The metric notions distance, diameter, width refer to the metric induced by the norm. Notions like 'convex', 'starshaped' in the space $\mathcal{K}^{n}$ refer to Minkowski linear combinations, defined with the aid of Minkowski addition of convex bodies and multiplication with nonnegative scalars.

Since it suffices to study complete sets in $X$ only up to dilatations, we define $D_{2}$ as the set of all complete sets of diameter 2 (the diameter of the unit ball) in $X$. It is the structure of this subspace of $\mathcal{K}^{n}$ that interests us in the present paper.

The first natural question to ask is whether the set $D_{2}$ is convex. It certainly is convex in those spaces which, like Euclidean space, have the property that every complete set in the space is of constant width (this is property (A), in a terminology introduced by Eggleston [3]): if $K, L$ are convex bodies of constant width and of diameter 2 , then $(1-\lambda) K+\lambda L$ is of constant width and of diameter 2 , for $\lambda \in[0,1]$. Conversely, suppose that $D_{2}$ is convex. Then the proof of Proposition 3.1 in [12] shows that the space $X$ has property (A). It is known (Theorem 3 in [13] is a stronger result) that, for $n \geq 3$, most $n$-dimensional Minkowski spaces (in the Baire category sense) do not have property (A). Thus, $D_{2}$ is generally not convex.

The next natural question, whether $D_{2}$ is always starshaped, also has a negative answer. A simple example to this effect is given in Sect. 4 (Example 2). We show in Sect. 3 that $D_{2}$ is starshaped if and only if the space $X$ has the property, denoted by (F) in [13], that the set of all complete sets is closed under the operation of adding a unit ball. The result from [13] mentioned above states that, for $n \geq 3$, most $n$-dimensional Minkowski spaces do not have property (F).

Readers who by now are getting worried whether $D_{2}$ might even not be connected, can be comforted: we prove in Sect. 3 that $D_{2}$ is contractible.

Fairly satisfactory information on the structure of $D_{2}$ is available for polyhedral Minkowski spaces. As our main result, we prove in Sect. 4 that for spaces $X$ with a polytopal unit ball, the space $\mathcal{D}_{X}$ of translation classes of bodies in $D_{2}$ is the union set of a finite polytopal complex. 
We conclude, in Sect. 5, with some observations on the extreme elements of $D_{2}$, that is, those elements which are not a non-trivial convex combination of two other elements of $D_{2}$.

\section{Preliminaries}

Recall that a bounded set $K$ in $X$ is diametrically complete, or briefly complete, if $\operatorname{diam}(K \cup\{x\})>\operatorname{diam} K$ for all $x \in \mathbb{R}^{n} \backslash K$.

The complete sets have a useful characterization in terms of supporting slabs. A supporting slab of the convex body $K \in \mathcal{K}^{n}$ is any closed set $\Sigma \supset K$ that is bounded by two parallel supporting hyperplanes $H, H^{\prime}$ of $K$. The distance between $H$ and $H^{\prime}$ is denoted by $w(\Sigma)$ and called the width of $\Sigma$. If $M$ is another convex body, we say that the supporting slab $\Sigma$ of $K$ is $M$-regular if the supporting slab of $M$ that is parallel to $\Sigma$ has the property that at least one of its bounding hyperplanes contains a smooth boundary point of $M$ (that is, a boundary point through which passes only one supporting hyperplane of $M)$. We use this terminology only in the cases where $M$ is one of the bodies $K, B, K+\alpha B(\alpha>0)$. It is not difficult to show that the diameter of the convex body $K$ is the supremum of the widths of all $B$-regular supporting slabs of $K$ (see the Remark before Theorem 1 in [13]). Hence, $K$ has diameter $d$ if and only if all $B$-regular supporting slabs $\Sigma$ of $K$ have width $w(\Sigma) \leq d$ and at least one supporting slab of $K$ has width $d$. For a convex body $K$ (with interior points) of diameter $d$, it is stated in [13, Theorem 1], that $K$ is complete if and only if every $K$-regular supporting slab of $K$ has width $d$. We collect these results in the following lemma.

Lemma 1 Let $d>0$. The $n$-dimensional convex body $K \in \mathcal{K}^{n}$ is a complete set of diameter $d$ if and only if the following properties hold:

(a) Every $B$-regular supporting slab of $K$ has width $\leq d$.

(b) Every $K$-regular supporting slab of $K$ has width $d$.

Back to $X$, we recall further that a completion of the convex body $K \in \mathcal{K}^{n}$ is a complete set that contains $K$ and has the same diameter as $K$. Every convex body $K \in \mathcal{K}^{n}$ has a completion; this is in general not unique. We denote the set of all completions of $K$ by $\gamma(K)$. This defines a mapping $\gamma: \mathcal{K}^{n} \rightarrow \mathcal{C}\left(\mathcal{K}^{n}\right)$, the diametric completion mapping (see [12]). Here $\mathcal{C}\left(\mathcal{K}^{n}\right)$ denotes the set of nonempty compact subsets of $\mathcal{K}^{n}$. In [12], the set $\mathcal{K}^{n}$ was metrized by the Hausdorff metric $\rho$ induced by the norm, and $\mathcal{C}\left(\mathcal{K}^{n}\right)$ was metrized by the Hausdorff metric induced by the metric $\rho$. Then it was shown that the diametric completion mapping is locally Lipschitz continuous. This was used to show that in spaces $X$ with a strictly convex norm the diametric completion mapping has a continuous selection. Without the assumption of strict convexity, we can show that there is a continuous mapping $\tau: \mathcal{K}^{n} \rightarrow \mathcal{K}^{n}$ such that some translate of $\tau(K)$ is a completion of $K$, for all $K \in \mathcal{K}^{n}$. This is part of the proof of Theorem 2 below. 


\section{Starshapedness and Connectedness}

Let $Z \in D_{2}$. By definition, the set $D_{2}$ is starshaped with respect to $Z$ if for any convex body $K \in D_{2}$ the segment $\{(1-\lambda) K+\lambda Z: 0 \leq \lambda \leq 1\}$ belongs to $D_{2}$. The set $D_{2}$ is said to be starshaped if there exists some $Z \in D_{2}$ with respect to which it is starshaped.

Lemma 2 If $D_{2}$ is starshaped, then $D_{2}$ is also starshaped with respect to the ball $B$.

Proof Let $D_{2}$ be starshaped with respect to $Z \in D_{2}$. Then $D_{2}$ is also starshaped with respect to $-Z$, since $K \in D_{2}$ implies $-K \in D_{2}$.

Since $D_{2}$ is starshaped with respect to $Z$, the segment joining $-Z$ to $Z$ belongs to $D_{2}$, in particular, $(1 / 2) Z+(1 / 2)(-Z) \in D_{2}$. Since the body $(1 / 2) Z+(1 / 2)(-Z)$ is complete (of diameter 2 ) and $o$-symmetric, it is the ball $B$, as is well known and easy to see. Now let $K \in D_{2}$ be given and let $C=(1-\lambda) K+\lambda B$ with $\lambda \in[0,1]$. We put

$$
\alpha:=\frac{2-\lambda}{2}, \quad \beta:=\frac{\lambda}{2-\lambda},
$$

then $\alpha, \beta \in[0,1]$. Let $M:=(1-\beta) K+\beta(-Z)$. Since $D_{2}$ is starshaped with respect to $-Z$, we have $M \in D_{2}$. Using $(1 / 2) Z+(1 / 2)(-Z)=B$, we compute that $C=$ $(1-\alpha) Z+\alpha M$, which shows that $C \in D_{2}$. We have proved that $\{(1-\lambda) K+\lambda B$ : $0 \leq \lambda \leq 1\} \subset D_{2}$, thus $D_{2}$ is starshaped with respect to $B$.

It is convenient in the following to use, as an auxiliary tool, also the Euclidean structure induced by the standard scalar product of $\mathbb{R}^{n}$. In this way, we can talk of normal cones and of the Steiner point, for example, and also use the Euclidean volume.

Lemma 3 Let $K \in \mathcal{K}^{n}$ be a convex body of diameter $d>0$. For any $\alpha>0$, the following assertions are equivalent:

(a) $K+\alpha B$ is complete.

(b) Every $(K+B)$-regular supporting slab of $K$ has width $d$.

Proof Clearly, $\operatorname{diam}(K+\alpha B) \leq d+2 \alpha$. The body $K$ has a supporting slab of width $d$. Since the widths of parallel supporting slabs are additive under Minkowski addition, and every supporting slab of $\alpha B$ has width $2 \alpha$, it follows that $K+\alpha B$ has some supporting slab of width $d+2 \alpha$ and hence has diameter $d+2 \alpha$.

Suppose that (a) holds. By Lemma 1, every $(K+\alpha B)$-regular supporting slab of $K+\alpha B$ has width $d+2 \alpha$. It follows that every $(K+\alpha B)$-regular supporting slab of $K$ has width $d$.

Let $\Sigma$ be a $(K+B)$-regular supporting slab of $K$. Then there is a supporting hyperplane $H$ of $K+B$ that is parallel to $\Sigma$ and contains a smooth boundary point $z$ of $K+B$. The normal cone $N(K+B, z)$ is of dimension one. We have $z=x+y$ with suitable points $x \in K$ and $y \in B$, lying in supporting hyperplanes of $K$ and $B$, respectively, parallel to $H$. By Theorem 2.2.1(a) of [14],

$$
N(K+B, z)=N(K, x) \cap N(B, y) .
$$


Since $N(\alpha B, \alpha y)=N(B, y)$, we get

$$
N(K+\alpha B, x+\alpha y)=N(K, x) \cap N(\alpha B, \alpha y)=N(K+B, z),
$$

which is of dimension one. It follows that $x+\alpha y$ is a smooth boundary point of $K+\alpha B$. Thus, the supporting slab $\Sigma$ is $(K+\alpha B)$-regular and hence has width $d$. This proves (b).

Suppose that (b) holds. Let $\Sigma$ be a $(K+\alpha B)$-regular supporting slab of $K+$ $\alpha B$. By a similar argument as used above, $\Sigma$ is also $(K+B)$-regular, hence the parallel supporting slab of $K$ has width $d$, by (b). Therefore, $\Sigma$ has width $d+2 \alpha$. By Lemma $1, K+\alpha B$ is complete.

The essential point of Lemma 3 is that condition (b) is independent of $\alpha$. Therefore, the completeness of $K+\alpha B$ for one positive number $\alpha>0$ implies the completeness of $K+\alpha B$ for all positive numbers $\alpha>0$ (and hence also the completeness of $K$ ).

We recall from [13] that the space $X$ is said to have property (F) if, for any complete set $K$, also the Minkowski sum $K+B$ is complete.

Theorem 1 The set $D_{2}$ is starshaped if and only if the space $X$ has property $(\mathrm{F})$.

Proof If $X$ has property (F), then it follows from Lemma 3 that, for any $K \in D_{2}$ and any $\lambda \in[0,1]$, the set $(1-\lambda) K+\lambda B$ is complete. By Lemma 1 , it has diameter 2 . Thus, $D_{2}$ is starshaped with respect to $B$.

If $D_{2}$ is starshaped, then it is starshaped with respect to $B$, by Lemma 2. Any complete set $K$ of positive diameter has a homothet $K^{\prime} \in D_{2}$, and then $(1 / 2) K^{\prime}+$ $(1 / 2) B$ is in $D_{2}$ and hence complete. Using Lemma 3 , we conclude that $K+B$ is complete. Thus, $X$ has property $(\mathrm{F})$.

Theorem 2 The space $D_{2}$ is contractible.

Proof As in [12], we use an idea of Groemer [5]. He called a set $M \in \mathcal{K}^{n}$ a tight cover of $K \in \mathcal{K}^{n}$ if $K \subset M$ and diam $M=\operatorname{diam} K$. He proved that among all tight covers of $K$ there is at least one which has maximal volume and that this is a completion of $K$. We call any such completion a maximal volume completion, briefly mv-completion, of $K$ and denote by $\gamma_{\mathrm{mv}}(K)$ the set of all mv-completions of $K$. For strictly convex norms, Groemer has shown that there is only one mv-completion of $K$, but without that assumption, we must work with the set $\gamma_{\mathrm{mv}}(K)$. (The simplest example for nonunique mv-completions is provided by the space $\ell_{\infty}^{2}$ and a segment $K$ which is not parallel to one of the diagonals of the unit ball, a parallelogram.)

Let $K \in \mathcal{K}^{n}$. As shown by Groemer with the aid of the Brunn-Minkowski theorem, any two elements of $\gamma_{\mathrm{mv}}(K)$ are translates of each other. Hence, if we choose any $M \in \gamma_{\mathrm{mv}}(K)$ and put

$$
\tau(K):=M-s(M)
$$

where $s(M)$ denotes the Steiner point of $M$ (with respect to the auxiliary Euclidean structure), then $\tau(K)$ does not depend on the choice of $M$. (The Steiner point mapping could be replaced by any continuous mapping from $\mathcal{K}^{n}$ to $\mathbb{R}^{n}$.) This defines a 
mapping $\tau: \mathcal{K}^{n} \rightarrow \mathcal{K}^{n}$. To prove the continuity of $\tau$, we modify the proof of Theorem 2.1 in [12]. Denoting by $V$ the volume we define, for $K \in \mathcal{K}^{n}$,

$$
\psi(K):=\max \{V(M): M \in \gamma(K)\} .
$$

Let $K_{i}, K \in \mathcal{K}^{n}$ be such that $\lim _{i \rightarrow \infty} K_{i}=K$. We will prove that $\lim _{i \rightarrow \infty} \tau\left(K_{i}\right)=$ $\tau(K)$ by showing that every subsequence of $\left(\tau\left(K_{i}\right)\right)_{i \in \mathbb{N}}$ has a further subsequence that converges to $\tau(K)$. There is no loss of generality if we assume, to simplify the notation, that the first subsequence is the original sequence $\left(\tau\left(K_{i}\right)\right)_{i \in \mathbb{N}}$. For each $i \in$ $\mathbb{N}$, let $M_{i} \in \gamma_{\mathrm{mv}}\left(K_{i}\right)$. The sequence $\left(M_{i}\right)_{i \in \mathbb{N}}$ is bounded and hence, by the Blaschke selection theorem, has a subsequence $\left(M_{i_{j}}\right)_{j \in \mathbb{N}}$ that converges to a convex body $M$. Since $M_{i_{j}}$ is a completion (and therefore a tight cover) of $K_{i j}$, the body $M$ is a tight cover of $K$. It was shown in the proof of Theorem 2.1 in [12] that $\psi$ (which was called $\varphi \circ \gamma$ in that proof) is continuous. It follows that

$$
V(M)=\lim _{j \rightarrow \infty} V\left(M_{i_{j}}\right)=\lim _{j \rightarrow \infty} \psi\left(K_{i_{j}}\right)=\psi(K)
$$

and thus $M \in \gamma_{\mathrm{mv}}(K)$, since $\psi(K) \geq V(L)$ whenever $L$ is a tight cover of $K$. Using now that the Steiner point mapping is continuous, we deduce that

$$
\lim _{j \rightarrow \infty} \tau\left(K_{i_{j}}\right)=\lim _{j \rightarrow \infty}\left(M_{i_{j}}-s\left(M_{i_{j}}\right)\right)=M-s(M)=\tau(K) .
$$

Finally, for $K \in D_{2}$ and $\lambda \in[0,1]$, let $K_{\lambda}:=(1-\lambda) K+\lambda B$ and

$$
F(K, \lambda):=\tau\left(\frac{2}{\operatorname{diam} K_{\lambda}} K_{\lambda}\right)+(1-\lambda) s(K)+\lambda s(B) .
$$

This defines a mapping $F: D_{2} \times[0,1] \rightarrow D_{2}$, which satisfies $F(K, 0)=K$ and $F(K, 1)=B$, since $K$ and $B$ are complete and of diameter 2 . By the continuity of the mapping $(K, \lambda) \mapsto K_{\lambda}$, of $\tau$ and of diameter and Steiner point, the mapping $F$ is continuous. Hence, the space $D_{2}$ is contractible.

\section{Polyhedral Norms}

In this section, we consider complete sets only up to translations. For $K \in \mathcal{K}^{n}$, the set of all translates $K+t$ of $K$, where $t \in \mathbb{R}^{n}$, is denoted by [K], and [ $\left.\mathcal{K}^{n}\right]$ is the space of all these translation classes, with the quotient topology. The Minkowski operations of addition and nonnegative scalar multiplication carry over from $\mathcal{K}^{n}$ to $\left[\mathcal{K}^{n}\right]$, by means of the definitions $[K]+[L]:=[K+L]$ and $\lambda[K]:=[\lambda K]$ for $K, L \in \mathcal{K}^{n}$ and $\lambda \geq 0$. Therefore, the usual convexity notions, like segment, starshaped, convex combination, convex set, extreme point, convex hull, polytope, etc., make sense in $\left[\mathcal{K}^{n}\right]$. A mapping $\varphi: C \rightarrow\left[\mathcal{K}^{n}\right]$ from a convex subset $C$ of a real vector space into $\left[\mathcal{K}^{n}\right]$ is Minkowski linear if $\varphi((1-\lambda) x+\lambda y)=(1-\lambda) \varphi(x)+\lambda \varphi(y)$ for all $x, y \in C$ and all $\lambda \in[0,1]$.

We denote by $\mathcal{D}_{X}$ the set of translation classes of the bodies in $D_{2}$. Then $\mathcal{D}_{X}$ is a compact subset of $\left[\mathcal{K}^{n}\right]$. It need not be convex, but it still makes sense to consider its extreme points. The element $[K]$ of $\mathcal{D}_{X}$ is called an extreme point of $\mathcal{D}_{X}$ if a representation $[K]=(1-\lambda)\left[K_{1}\right]+\lambda\left[K_{2}\right]$ with $K_{1}, K_{2} \in \mathcal{D}_{X}$ and $0<\lambda<1$ is only possible with $\left[K_{1}\right]=\left[K_{2}\right]=[K]$. The set $\mathcal{D}_{X}$ is our object of study in the present 
section, for the case where the space $X=\left(\mathbb{R}^{n},\|\cdot\|\right)$ is polyhedral, that is, the unit ball $B$ is a polytope. This is assumed from now on.

The following lemma leads to an intuitive description of $\mathcal{D}_{X}$ in simple cases.

Lemma 4 Let $\Sigma_{1}, \ldots, \Sigma_{k}$ be the B-regular supporting slabs of the polytopal unit ball $B$. Each $K \in D_{2}$ is of the form

$$
K=\bigcap_{i=1}^{k}\left(\Sigma_{i}+t_{i}\right)
$$

with $t_{i} \in \mathbb{R}^{n}, i=1, \ldots, k$. Conversely, if $K \neq \emptyset$ is given by (1), then $K \in D_{2}$ if and only if each $\Sigma_{i}+t_{i}$ that is $K$-regular is a supporting slab of $K$.

Proof Let $\pm u_{1}, \ldots, \pm u_{k}$ be the outer unit normal vectors of the facets of $B$, and denote by $H^{-}(K, u)$ the supporting halfspace of $K$ with outer normal vector $u$. If $K \in D_{2}$, then $K=\bigcap_{x \in K}(2 B+x)$ (the spherical intersection property, see Eggleston [3]), therefore

$$
K=\bigcap_{i=1}^{k}\left(H^{-}\left(K, u_{i}\right) \cap H^{-}\left(K,-u_{i}\right)\right) .
$$

The bounding hyperplanes $H_{i}$ of $H^{-}\left(K, u_{i}\right)$ and $H_{i}^{\prime}$ of $H^{-}\left(K,-u_{i}\right)$ are at distance at most 2 apart, since $K \in D_{2}$. If their distance is equal to 2 , then $H^{-}\left(K, u_{i}\right) \cap$ $H^{-}\left(K,-u_{i}\right)=\Sigma_{i}+t_{i}$ for a suitable vector $t_{i}$. If their distance is less than 2 , then by Lemma 1 none of the hyperplanes $H_{i}, H_{i}^{\prime}$ contains a facet of the polytope $K$, and in (2) we can replace $H^{-}\left(K, u_{i}\right) \cap H^{-}\left(K,-u_{i}\right)$ by $\Sigma_{i}+t_{i}$ for a suitable vector $t_{i}$, without changing the intersection. Thus, $K$ can be represented in the form (1). Conversely, let $K \neq \emptyset$ be given by (1). Then each $B$-regular supporting slab of $K$ has width at most 2, and each $K$-regular supporting slab of $K$ is parallel to one of the slabs $\Sigma_{1}, \ldots, \Sigma_{k}$. The assertion now follows from Lemma 1 .

To obtain representatives of the classes in $\mathcal{D}_{X}$, that is, elements of $D_{2}$ up to translations, we may fix $n$ of the $B$-regular supporting slabs of $B$ with linearly independent normal vectors and translate only the remaining ones, to obtain representations of the form (1).

Example 1 Let $X=\ell_{1}^{3}$, that is, $\mathbb{R}^{n}$ with the norm with unit ball given by the convex hull of the points $\pm(1,0,0), \pm(0,1,0), \pm(0,0,1)$. Its supporting slabs are $\Sigma_{1}$ with outer normal vectors $\pm(1,1,1), \Sigma_{2}$ with outer normal vectors $\pm(-1,1,1), \Sigma_{3}$ with outer normal vectors $\pm(1,-1,1), \Sigma_{4}$ with outer normal vectors $\pm(-1,-1,1)$. We fix $\Sigma_{2}, \Sigma_{3}, \Sigma_{4}$, then each translation class of $\mathcal{D}_{X}$ has a representative of the form

$$
P(\alpha):=\Sigma_{2} \cap \Sigma_{3} \cap \Sigma_{4} \cap\left(\Sigma_{1}+\alpha(1,1,1)\right)
$$

with suitable $\alpha \in \mathbb{R}$. Precisely for $|\alpha| \leq 2 / 3$ the condition of Lemma 4 is satisfied, that is, $P_{\alpha}$ is an element of $D_{2}$. Moreover, the mapping $\alpha \mapsto[P(\alpha)]$ is Minkowski linear on the interval $[0,2 / 3]$ and on the interval $[-2 / 3,0]$, but on no larger interval. 

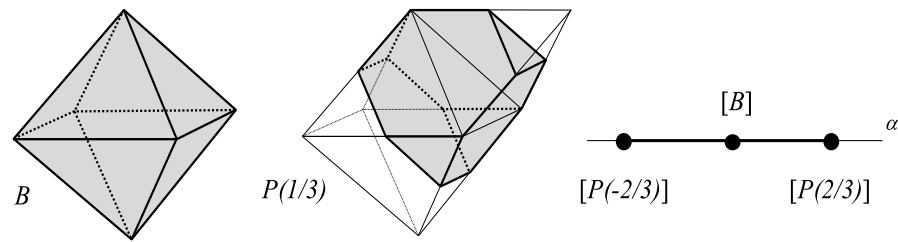

Fig. 1 Diametrically complete sets in $\ell_{1}^{3}$

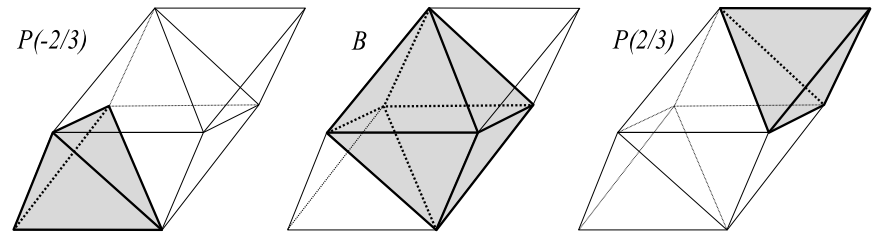

Fig. 2 The extreme points of $\mathcal{D}_{X}$ in $\ell_{1}^{3}$
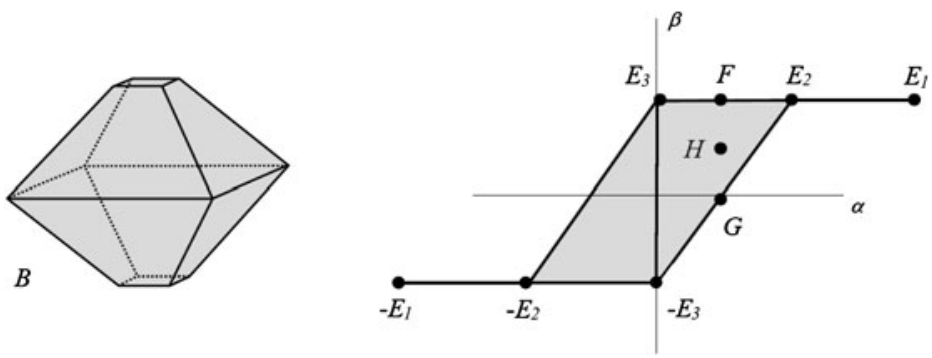

Fig. 3 Structure of $\mathcal{D}_{X}$ for a special polyhedral norm

Thus, $\mathcal{D}_{X}$ consists of two closed segments with common endpoint $[B]$, as indicated in the right part of Fig. 1.

We see that, in this simple example, $\mathcal{D}_{X}$ is not convex, but is still starshaped. It has got three extreme points, namely $[B],\left[P\left(\frac{2}{3}\right)\right],\left[P\left(-\frac{2}{3}\right)\right]$. They are shown in Fig. 2 .

The following is perhaps the simplest example where $\mathcal{D}_{X}$ is not starshaped, and it gives a hint what to expect of $\mathcal{D}_{X}$ in the case of a general polyhedral norm.

Example 2 Let $X=\left(\mathbb{R}^{3},\|\cdot\|\right)$ with the norm determined by the following unit ball $B$. Let $B$ be obtained from the octahedron with vertices $\pm(1,0,0), \pm(0,1,0), \pm(0,0,1)$, by cutting off the vertices $\pm(0,0,1)$ by the planes orthogonal to $(0,0,1)$ and at $(\mathrm{Eu}-$ clidean) distance 3/4 from the origin (see Fig. 3). The supporting slabs of $B$ are $\Sigma_{1}, \ldots, \Sigma_{4}$ from Example 1 and $\Sigma_{5}$, with normal vectors $\pm(0,0,1)$.

Again we fix $\Sigma_{2}, \Sigma_{3}, \Sigma_{4}$, then each translation class of $\mathcal{D}_{X}$ has a representative of the form

$$
P(\alpha, \beta):=\Sigma_{2} \cap \Sigma_{3} \cap \Sigma_{4} \cap\left(\Sigma_{1}+\alpha(1,1,1)\right) \cap\left(\Sigma_{5}+\beta(0,0,1)\right)
$$



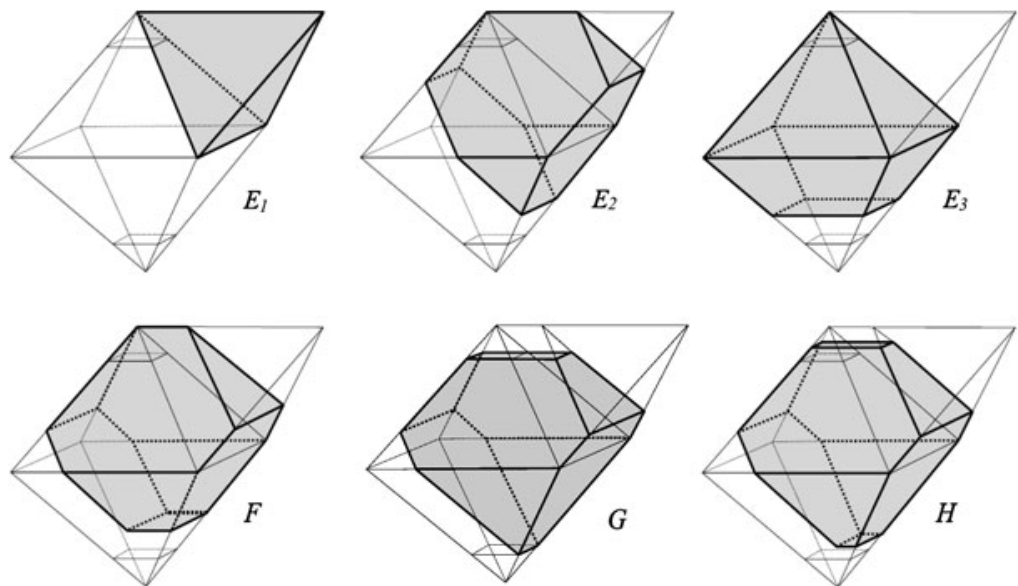

Fig. 4 Different complete sets for the norm of Fig. 3

with suitable numbers $\alpha, \beta \in \mathbb{R}$. In this case we find that $\mathcal{D}_{X}$ has precisely the six extreme points $\left[ \pm E_{1}\right],\left[ \pm E_{2}\right],\left[ \pm E_{3}\right]$, where $E_{1}=P\left(\frac{2}{3}, \frac{1}{4}\right), E_{2}=P\left(\frac{1}{3}, \frac{1}{4}\right)$ and $E_{3}=$ $P\left(0, \frac{1}{4}\right)$ (see Fig. 4).

As indicated in the right part of Fig. 3, the set $\mathcal{D}_{X}$ consists of the following four convex polytopes of translation classes: $\operatorname{conv}\left\{\left[E_{1}\right],\left[E_{2}\right]\right\}, \operatorname{conv}\left\{\left[-E_{1}\right],\left[-E_{2}\right]\right\}$, $\operatorname{conv}\left\{\left[E_{2}\right],\left[E_{3}\right],\left[-E_{3}\right]\right\}, \operatorname{conv}\left\{\left[-E_{2}\right],\left[-E_{3}\right],\left[E_{3}\right]\right\}$. Note that neither $\left[E_{1}\right]+\left[-E_{1}\right]$ nor $\left[E_{2}\right]+\left[-E_{2}\right]$ is complete, since they are not intersections of balls. There is no element of $\mathcal{D}_{X}$ to which each of $\left[E_{1}\right]$ and $\left[-E_{1}\right]$ could be connected by a segment, hence $\mathcal{D}_{X}$ is not starshaped.

Figure 4 shows three of the extreme points of $\mathcal{D}_{X}$, two points in the relative interiors of one-dimensional faces, and one point in the interior of a two-dimensional face of $\mathcal{D}_{X}$.

The main purpose of this section is to show that for a polyhedral Minkowski space, the set $\mathcal{D}_{X}$ is always the union set of a finite polytopal complex. In particular, $\mathcal{D}_{X}$ is the union of finitely many convex sets, and it has only finitely many extreme points.

Recall that a polyhedral complex in some $\mathbb{R}^{d}$ is a family $\mathcal{C}$ of convex polyhedral sets in $\mathbb{R}^{d}$ with the property that the intersection of any two polyhedra in $\mathcal{C}$ is either empty or a polyhedron of $\mathcal{C}$ and that any face of a polyhedron in $\mathcal{C}$ belongs to $\mathcal{C}$. A polytopal complex is a polyhedral complex all of whose polyhedra are bounded. We call a family $\mathcal{F}$ of sets in $\left[\mathcal{K}^{n}\right]$ a polytopal complex if there are a polytopal complex $\mathcal{C}$ in some $\mathbb{R}^{d}$ and a mapping $\varphi: \mathbb{R}^{d} \rightarrow\left[\mathcal{K}^{n}\right]$ such that $\mathcal{F}=\{\varphi(P): P \in \mathcal{C}\}$ and the restriction of $\varphi$ to any polytope of $\mathcal{C}$ is Minkowski linear.

In Example 2, we can take $d=2$ and for $\mathcal{C}$ the complex consisting of the polytopes $\pm Q_{1}, Q_{2}, Q_{3}$ in $\mathbb{R}^{2}$ given by

$$
\begin{aligned}
& Q_{1}:=\operatorname{conv}\left\{\left(\frac{1}{3}, \frac{1}{4}\right), \pm\left(0, \frac{1}{4}\right)\right\}, \quad Q_{2}:=\operatorname{conv}\left\{\left(\frac{2}{3}, \frac{1}{4}\right),\left(\frac{1}{3}, \frac{1}{4}\right)\right\}, \\
& Q_{3}:=-Q_{2}
\end{aligned}
$$

and their faces, and for $\varphi$ the mapping $(\alpha, \beta) \mapsto[P(\alpha, \beta)]$. 
Theorem 3 If $X$ is a polyhedral Minkowski space, then $\mathcal{D}_{X}$ is the union set of a finite polytopal complex.

The proof of the theorem requires a number of preparations. As already mentioned, it is convenient to use the standard Euclidean scalar product $\langle\cdot, \cdot\rangle$ as an auxiliary tool. This allows us to use Euclidean normal vectors of hyperplanes and, later, to employ the support function $h_{K}$ of $K \in \mathcal{K}^{n}$, as a function on $\mathbb{R}^{n}$. In particular, a closed halfspace can be written in the form

$$
H^{-}(u, \eta):=\left\{x \in \mathbb{R}^{n}:\langle x, u\rangle \leq \eta\right\}
$$

with $u \in \mathbb{R}^{n} \backslash\{o\}$ and $\eta \in \mathbb{R}$.

We shall deduce Theorem 3 from a result of McMullen [10], which is based on the technique of diagrams and representations of polyhedra. We refer to [10] for this technique, but we must explain McMullen's approach with the necessary details that we need to apply his result.

Let $\pm u_{1}, \ldots, \pm u_{N}$ be the outer unit normal vectors of the facets of the unit ball $B$. We write $U$ for the $k$-tuple $\left(u_{1}, \ldots, u_{k}\right), k=2 N$, with $u_{i+N}:=-u_{i}$ for $i=1, \ldots, N$. Let $\sigma$ be a representation associated with $U$, that is, a linear mapping from $\mathbb{R}^{k}$ into $\mathbb{R}^{k-n}$ with kernel

$$
\operatorname{ker} \sigma=\left\{\left(\left\langle b, u_{1}\right\rangle, \ldots,\left\langle b, u_{k}\right\rangle\right): b \in \mathbb{R}^{n}\right\} .
$$

We refer to [10] for the construction of such a mapping by means of a 'linear representation'. If $\left(e_{1}, \ldots, e_{k}\right)$ is the standard basis of $\mathbb{R}^{k}$, we define $\bar{u}_{i}:=\sigma\left(e_{i}\right)$ for $i=1, \ldots, k$ and denote by $\bar{U}$ the $k$-tuple $\left(\bar{u}_{1}, \ldots, \bar{u}_{k}\right)$. The $k$-tuple $\bar{U}$ is called a representation of $U$.

For given $\left(\eta_{1}, \ldots, \eta_{k}\right) \in \mathbb{R}^{k}$, the set

$$
P:=\bigcap_{i=1}^{k} H^{-}\left(u_{i}, \eta_{i}\right)
$$

is either empty or a polytope whose facets have only normal vectors from $\left\{u_{1}, \ldots, u_{k}\right\}$. The set of all polytopes of the form (4) is denoted by $\mathcal{P}(U)$. If $P \neq \emptyset$, the point

$$
p=\sigma\left(\eta_{1}, \ldots, \eta_{k}\right)=\sum_{i=1}^{k} \eta_{i} \bar{u}_{i}
$$

is said to be associated with $P$. In virtue of (3), the point $p$ is associated precisely with the polytopes of the translation class [P].

The following results are proved in [10]. Let $P \in \mathcal{P}(U)$. Then $p \in \operatorname{pos} \bar{U}$, and int $P \neq \emptyset$ if and only if $p \in \operatorname{int} \operatorname{pos} \bar{U}$. The vector $u_{i}$ is the normal vector of some facet of $P$ if and only if $p \in$ int pos $\left(\bar{U} \backslash\left\{\bar{u}_{i}\right\}\right)$.

For a given $P \in \mathcal{P}(U)$, the numbers $\eta_{i}$ in (4) are in general not uniquely determined by $P$, but we can always assume that

$$
\eta_{i}=\eta_{i}(P):=h\left(P, u_{i}\right)
$$

for $i=1, \ldots, k$; here $h(P, \cdot)$ is the support function of $P$. The point $p(P)=$ $\sum_{i=1}^{k} h\left(P, u_{i}\right) \bar{u}_{i}$ is called the representative of $P$. All translates of $P$ have the same 
representative. A point $p$ associated with $P$ is its representative if and only if it belongs to the closed inner region, which is defined by

$$
\operatorname{clir} \bar{U}:=\bigcap_{j=1}^{k} \operatorname{pos}\left(\bar{U} \backslash\left\{\bar{u}_{j}\right\}\right) .
$$

The continuous, surjective mapping

$$
\varrho: \quad \mathcal{P}(U) \rightarrow \operatorname{clir} \bar{U}, \quad P \mapsto p(P),
$$

which associates with each polytope of $\mathcal{P}(U)$ its representative, has the property that $\varrho^{-1}(p) \in \mathcal{P}_{T}(U)$ for $p \in \operatorname{clir} \bar{U}$, where $\mathcal{P}_{T}(U)$ (using McMullen's notation) denotes the set of translation classes of polytopes from $\mathcal{P}(U)$ (as a subspace of $\left[\mathcal{K}^{n}\right]$, hence with the quotient topology).

As defined by McMullen [10], a type-cone is any subset of clir $\bar{U}$ which is a nonempty intersection of sets of the form relint pos $\widetilde{V}$ with $\widetilde{V} \subseteq \bar{U}$. Thus, $\operatorname{clir} \bar{U}$ is partitioned into the type-cones. The bijective mapping

$$
\varrho^{-1}: \quad \operatorname{clir} \bar{U} \rightarrow \mathcal{P}_{T}(U)
$$

has the following properties. It is continuous. Restricted to the closure of any typecone, it is Minkowski linear. And, most important: points $p$ and $q$ of clir $\bar{U}$ belong to the same type-cone if and only if their images under $\varrho^{-1}$ are translation classes of strongly isomorphic polytopes. The polytopes $P_{1}, P_{2}$ are strongly isomorphic (see $[14$, pp. $100 \mathrm{ff}])$ if

$$
\operatorname{dim} F\left(P_{1}, u\right)=\operatorname{dim} F\left(P_{2}, u\right)
$$

for all vectors $u \neq o$; here $F(P, u)$ denotes the support set of $P$ with outer normal vector $u$. This is an equivalence relation, and the equivalence class of a polytope $P$ is called its a-type. (The 'a' comes from 'analogous', which is used synonymously for 'strongly isomorphic.)

Theorem 6 of [10] characterizes the type-cones as the maximal relatively open convex subsets of clir $\bar{U}$ on which $\varrho^{-1}$ is Minkowski linear. Finally, Theorem 8 of [10] states that the closures of the type-cones in clir $\bar{U}$ form a polyhedral complex.

Now we proceed to the proof of Theorem 3.

Lemma 5 For each type-cone $\mathcal{K}$, the intersection $\mathcal{K} \cap \varrho\left(D_{2}\right)$ is convex.

Proof Let $\mathcal{K}$ be a type-cone, and suppose that $p, p^{\prime} \in \mathcal{K} \cap \varrho\left(D_{2}\right)$. Choose polytopes $P \in \varrho^{-1}(p)$ and $P^{\prime} \in \varrho^{-1}\left(p^{\prime}\right)$. Then

$$
p=\sum_{i=1}^{k} h\left(P, u_{i}\right) \bar{u}_{i}, \quad p^{\prime}=\sum_{i=1}^{k} h\left(P^{\prime}, u_{i}\right) \bar{u}_{i} .
$$

Let $\lambda \in[0,1]$ and put $Q:=(1-\lambda) P+\lambda P^{\prime}$. Then $q=(1-\lambda) p+\lambda p^{\prime}$ is the representative of $Q$.

We may assume, after renumbering, that $\pm u_{1}, \ldots, \pm u_{m}$ (for some $m \leq N$ ) are precisely the normal vectors of the $P$-regular supporting slabs of $P$. Recall that the supporting slab of $P$ with normal vector $u$ is $P$-regular if and only if 
$\max \{\operatorname{dim} F(P, u), \operatorname{dim} F(P,-u)\}=n-1$. Since $p$ and $q$ lie in the same typecone $\mathcal{K}$, the polytopes $P$ and $Q$ are strongly isomorphic, hence

$$
\operatorname{dim} F\left(Q, u_{i}\right)=\operatorname{dim} F\left(P, u_{i}\right),
$$

for $i=1, \ldots, k$. Therefore, $\pm u_{1}, \ldots, \pm u_{p}$ are precisely the normal vectors of the $Q$-regular supporting slabs of $Q$ and, of course, also of $P^{\prime}$.

Since $p, p^{\prime} \in \varrho\left(D_{2}\right)$, the polytopes $P, P^{\prime}$ are complete and have diameter 2 . Therefore, Lemma 1 gives

$$
h\left(P, u_{i}\right)+h\left(P,-u_{i}\right) \leq 2 h\left(B, u_{i}\right), \quad h\left(P^{\prime}, u_{i}\right)+h\left(P^{\prime},-u_{i}\right) \leq 2 h\left(B, u_{i}\right)
$$

for $i=1, \ldots, N$ and

$$
h\left(P, u_{i}\right)+h\left(P,-u_{i}\right)=2 h\left(B, u_{i}\right), \quad h\left(P^{\prime}, u_{i}\right)+h\left(P^{\prime},-u_{i}\right)=2 h\left(B, u_{i}\right)
$$

for $i=1, \ldots, m$. From (6) and (7) it follows that

$$
\begin{array}{ll}
h\left(Q, u_{i}\right)+h\left(Q,-u_{i}\right) \leq 2 h\left(B, u_{i}\right) & \text { for } i=1, \ldots, N, \\
h\left(Q, u_{i}\right)+h\left(Q,-u_{i}\right)=2 h\left(B, u_{i}\right) & \text { for } i=1, \ldots, m .
\end{array}
$$

We conclude that each $B$-regular supporting slab of $Q$ has width at most 2 and that each $Q$-regular supporting slab of $Q$ has width 2. By Lemma $1, Q$ has diameter 2 and is complete. Therefore, $q \in \varrho\left(D_{2}\right)$. Thus, $\mathcal{K} \cap \varrho\left(D_{2}\right)$ is convex.

Let $i \in\{1, \ldots, N\}$. The set

$$
H_{i}:=\left\{\left(\eta_{1}, \ldots, \eta_{k}\right) \in \mathbb{R}^{k}: \eta_{i}+\eta_{i+N}=2 h\left(B, u_{i}\right)\right\}
$$

is a hyperplane. Therefore, the set $\sigma\left(H_{i}\right)$ is a hyperplane in $\mathbb{R}^{k-n}$ (it is not the whole space, since $\left.H_{i} \cap \operatorname{ker} \sigma=\emptyset\right)$. Let $S_{i}^{+}, S_{i}^{-} \subset \mathbb{R}^{k-n}$ be the two open halfspaces bounded by $\sigma\left(H_{i}\right)$. Calling two points of $\mathbb{R}^{k-n}$ equivalent if they belong to the same elements of $\sigma\left(H_{1}\right), \ldots, \sigma\left(H_{N}\right), S_{1}^{+}, S_{1}^{-}, \ldots, S_{N}^{+}, S_{N}^{-}$, we obtain a partition of $\mathbb{R}^{k-n}$ into the equivalence classes. They are relatively open convex polyhedral sets and are called the cells. By a type-cell we understand any nonempty intersection of a type-cone and a cell. Since the closures of the type-cones form a polyhedral complex, by McMullen's result, and clearly also the closures of the cells form a polyhedral complex, we deduce that the closures of all type-cells form a polyhedral complex.

Lemma 6 Let $T$ be a type-cell, and suppose that $C:=T \cap \varrho\left(D_{2}\right)$ contains more than one point. Then

$$
\operatorname{relbd} C \subset \operatorname{relbd} T \text {. }
$$

Proof By Lemma 5, the set $C$ is convex. Suppose, contrary to the assertion, that there exists a point $p \in \operatorname{relbd} C$ with $p \in \operatorname{relint} T$. Since $C$ contains more than one point, we can choose a point $p^{\prime} \in \operatorname{relint} C$ with $p^{\prime} \neq p$. We can further choose a number $\epsilon>0$ such that $q_{\epsilon}:=p+\epsilon\left(p-p^{\prime}\right) \in T$, which is possible since $p \in \operatorname{relint} T$ and 
$p^{\prime} \in T$. Let $P \in \varrho^{-1}(p), P^{\prime} \in \varrho^{-1}\left(p^{\prime}\right)$ and $Q_{\epsilon} \in \varrho^{-1}\left(q_{\epsilon}\right)$. Since $p, p^{\prime}, q_{\epsilon} \in \operatorname{clir} \bar{U}$, we have

$$
p=\sum_{i=1}^{k} h\left(P, u_{i}\right) \bar{u}_{i}, \quad p^{\prime}=\sum_{i=1}^{k} h\left(P^{\prime}, u_{i}\right) \bar{u}_{i}, \quad q_{\epsilon}=\sum_{i=1}^{k} h\left(Q_{\epsilon}, u_{i}\right) \bar{u}_{i},
$$

hence

$$
\sum_{i=1}^{k}\left[\eta_{i}-h\left(Q_{\epsilon}, u_{i}\right)\right] \bar{u}_{i}=o \quad \text { with } \eta_{i}:=(1+\epsilon) h\left(P, u_{i}\right)-\epsilon h\left(P^{\prime}, u_{i}\right) .
$$

This implies

$$
\left(\eta_{1}-h\left(Q_{\epsilon}, u_{1}\right), \ldots, \eta_{k}-h\left(Q_{\epsilon}, u_{k}\right)\right) \in \operatorname{ker} \sigma .
$$

Therefore, there exists a vector $b \in \mathbb{R}^{n}$ with

$$
\eta_{i}-h\left(Q_{\epsilon}, u_{i}\right)=\left\langle b, u_{i}\right\rangle \quad \text { for } i=1, \ldots, k,
$$

which means that

$$
h\left(Q_{\epsilon}+b, u_{i}\right)=(1+\epsilon) h\left(P, u_{i}\right)-\epsilon h\left(P^{\prime}, u_{i}\right), \quad i=1, \ldots, k .
$$

Since $P, P^{\prime}, Q_{\epsilon}$ belong to the same type-cell, they belong to the same type-cone and hence are strongly isomorphic. As in the proof of Lemma 5, we may assume, after renumbering, that $\pm u_{1}, \ldots, \pm u_{m}$ are precisely the normal vectors of the $P$-regular supporting slabs of $P$; then $\pm u_{1}, \ldots, \pm u_{m}$ have the same meaning for $P^{\prime}$ and $Q_{\epsilon}$. Since $p, p^{\prime} \in \varrho\left(D_{2}\right)$, relations (6) and (7) are valid, and we deduce from (8) that

$$
h\left(Q_{\epsilon}, u_{i}\right)+h\left(Q_{\epsilon},-u_{i}\right)=2 h\left(B, u_{i}\right) \text { for } i=1, \ldots, m .
$$

Suppose that for some $j>m$ we have

$$
h\left(Q_{\epsilon}, u_{j}\right)+h\left(Q_{\epsilon},-u_{j}\right)>2 h\left(B, u_{j}\right) .
$$

We have $P \in D_{2}$ and hence $h\left(P, u_{j}\right)+h\left(P,-u_{j}\right) \leq 2 h\left(B, u_{j}\right)$. If equality holds here, then $p \in \sigma\left(H_{j}\right)$. Since $p, q_{\epsilon} \in T$, the points $p$ and $q_{\epsilon}$ are in the same typecell and hence belong to the same of the hyperplanes $\sigma\left(H_{1}\right), \ldots, \sigma\left(H_{N}\right)$, in particular $q_{\epsilon} \in \sigma\left(H_{j}\right)$, which implies that $h\left(Q_{\epsilon}, u_{j}\right)+h\left(Q_{\epsilon},-u_{j}\right)=2 h\left(B, u_{j}\right)$, a contradiction. Therefore, $h\left(P, u_{j}\right)+h\left(P,-u_{j}\right)<2 h\left(B, u_{j}\right)$. By continuity, there exists $\epsilon^{\prime}$ with $0 \leq \epsilon^{\prime} \leq \epsilon$ and $h\left(Q_{\epsilon^{\prime}}, u_{j}\right)+h\left(Q_{\epsilon^{\prime}},-u_{j}\right)=2 h\left(B, u_{j}\right)$. This means that $q_{\epsilon^{\prime}} \in \sigma\left(H_{j}\right)$, again a contradiction, for the same reason. Thus, the assumption was false, and we have $h\left(Q_{\epsilon}, u_{j}\right)+h\left(Q_{\epsilon},-u_{j}\right) \leq 2 h\left(B, u_{j}\right)$ for $j=1, \ldots, k$. This completes the proof of the fact that $Q_{\epsilon} \in D_{2}$ and hence $q_{\epsilon} \in \varrho\left(D_{2}\right)$. From $q_{\epsilon} \in C$ and $p^{\prime} \in$ relint $C$ and the convexity of $C$ it now follows that $p \in \operatorname{relint} C$, a contradiction. This completes the proof of Lemma 6.

By Lemma 6, the following holds. If $T \cap \varrho\left(D_{2}\right) \neq \emptyset$ for some type-cell $T$, then

$$
T \cap \varrho\left(D_{2}\right)=\operatorname{aff}\left(T \cap \varrho\left(D_{2}\right)\right) \cap T .
$$

It is now easy to see that the closures of the nonempty intersections $T \cap \varrho\left(D_{2}\right)$, where $T$ is a type-cell, form a polyhedral complex. It is, in fact, a polytopal complex, since $\mathcal{D}_{X}$ is compact and the inverse of the mapping $\varrho^{-1}$ is continuous. Since the mapping $\varrho^{-1}$ is Minkowski linear on each type-cone, and thus on each type-cell intersected with $\varrho\left(D_{2}\right)$, we have proved Theorem 3 . 


\section{Extreme Points}

In order to gather some more information about the space $\mathcal{D}_{X}$, we study its extreme points. First, we give an example in which any neighborhood of $[B]$ in $\mathcal{D}_{X}$ contains convex subsets of a given finite dimension, but $[B]$ itself is an extreme point.

The example is the space $X=\left(\mathbb{R}^{n},\|\cdot\|\right)$, where the unit ball $B$ of the norm is a polyhedral spindle, that is, a polytope with a vertex $p$ such that each facet of $B$ contains either $p$ or $-p$. Let $u_{1}, \ldots, u_{N}$ be the outer unit normal vectors of the facets of $B$ containing $p$, then $-u_{1}, \ldots,-u_{N}$ are the normal vectors of the facets containing $q:=-p$. Suppose that

$$
B=(1-\lambda) K_{0}+\lambda K_{1} \quad \text { with } K_{0}, K_{1} \in D_{2}, 0<\lambda<1 .
$$

For any vector $v \neq o$ with $F(B, v)=\{p\}$ it follows from (9) that $F\left(K_{0}, v\right)=\left\{p_{0}\right\}$, $F\left(K_{1}, v\right)=\left\{p_{1}\right\}$ with $p_{0} \in K_{0}, p_{1} \in K_{1}$ and $p=(1-\lambda) p_{0}+\lambda p_{1}$. Similarly, $F\left(K_{0},-v\right)=\left\{q_{0}\right\}, F\left(K_{1},-v\right)=\left\{q_{1}\right\}$ with $q_{0} \in K_{0}, q_{1} \in K_{1}$ and $q=(1-\lambda) q_{0}+$ $\lambda q_{1}$. For $i=1, \ldots, N$ we have

$$
\begin{aligned}
(1-\lambda) h\left(K_{0}, u_{i}\right)+\lambda h\left(K_{1}, u_{i}\right) & =\left\langle p, u_{i}\right\rangle=(1-\lambda)\left\langle p_{0}, u_{i}\right\rangle+\lambda\left\langle p_{1}, u_{i}\right\rangle \\
& \leq(1-\lambda) h\left(K_{0}, u_{i}\right)+\lambda h\left(K_{1}, u_{i}\right)
\end{aligned}
$$

hence $\left\langle p_{0}, u_{i}\right\rangle=h\left(K_{0}, u_{i}\right)$ and thus $p_{0} \in F\left(K_{0}, u_{i}\right)$, similarly $q_{0} \in F\left(K_{0},-u_{i}\right)$. Since $K_{0}, K_{1} \in D_{2}$, the Minkowski width $w$ satisfies $w\left(K_{0}, u_{i}\right) \leq 2, w\left(K_{1}, u_{i}\right) \leq 2$. From (9) we get

$$
2=w\left(B, u_{i}\right)=(1-\lambda) w\left(K_{0}, u_{i}\right)+\lambda w\left(K_{1}, u_{i}\right) \leq 2
$$

and hence $w\left(K_{0}, u_{i}\right)=2$. Therefore, $\left\langle p_{0}, u_{i}\right\rangle+\left\langle q_{0},-u_{i}\right\rangle=2\left\langle p, u_{i}\right\rangle$, which gives

$$
\left\langle p, u_{i}\right\rangle=\left\langle p_{0}, u_{i}\right\rangle-\left\langle\frac{1}{2}\left(p_{0}+q_{0}\right), u_{i}\right\rangle, \quad\left\langle q, u_{i}\right\rangle=\left\langle q_{0}, u_{i}\right\rangle-\left\langle\frac{1}{2}\left(p_{0}+q_{0}\right), u_{i}\right\rangle .
$$

Since

$$
\begin{aligned}
B & =\bigcap_{i=1}^{N}\left[H^{-}\left(u_{i},\left\langle p, u_{i}\right\rangle\right) \cap H^{-}\left(-u_{i},\left\langle q,-u_{i}\right\rangle\right)\right], \\
K_{0} & =\bigcap_{i=1}^{N}\left[H^{-}\left(u_{i},\left\langle p_{0}, u_{i}\right\rangle\right) \cap H^{-}\left(-u_{i},\left\langle q_{0},-u_{i}\right\rangle\right)\right],
\end{aligned}
$$

we now deduce that $B=K_{0}-\frac{1}{2}\left(p_{0}+q_{0}\right)$. Similarly, $K_{1}$ is a translate of $B$. This shows that $[B]$ is an extreme point of $\mathcal{D}_{X}$.

To show the second announced property of this example, let $\Sigma_{i}$ denote the $B$ regular supporting slab of $B$ with normal vectors $\pm u_{i}, i=1, \ldots, N$. We assume, without loss of generality, that $u_{N-n+1}, \ldots, u_{N}$ are linearly independent and consider the polytopes

$$
P\left(\alpha_{1}, \ldots, \alpha_{N-n}\right):=\bigcap_{i=1}^{N-n}\left(\Sigma_{i}+\alpha_{i} u_{i}\right) \cap \bigcap_{j=1}^{n} \Sigma_{N-n+j} .
$$


There is a neighborhood $U$ of $(0, \ldots, 0) \in \mathbb{R}^{N-n}$ such that for $\left(\alpha_{1}, \ldots, \alpha_{N-n}\right) \in U$ the polytope $P\left(\alpha_{1}, \ldots, \alpha_{N-n}\right)$ has the same number of facets as $B$ and belongs to $D_{2}$. Further, we can find $\left(\alpha_{1}^{0}, \ldots, \alpha_{N-n}^{0}\right) \in U$ such that the polytope $P\left(\alpha_{1}^{0}, \ldots, \alpha_{N-n}^{0}\right)$ is simple. By Lemma 2.4.12 of [14], there exists a neighborhood $V$ of $\left(\alpha_{1}^{0}, \ldots, \alpha_{N-n}^{0}\right)$ in $\mathrm{U}$ such that for $\left(\alpha_{1}, \ldots, \alpha_{N-n}\right) \in V$ the polytopes $P\left(\alpha_{1}, \ldots, \alpha_{N-n}\right)$ belong to the same equivalence class of strongly isomorphic polytopes. If $P_{1}, \ldots, P_{k}$ are strongly isomorphic polytopes in $D_{2}$, then the polytope $\lambda_{1} P_{1}+\cdots+\lambda_{k} P_{k}\left(\lambda_{1}, \ldots, \lambda_{k} \geq 0\right.$, $\lambda_{1}+\cdots+\lambda_{k}=1$ ) belongs to $D_{2}$ (as used in the proofs of Lemmas 5 and 6). We conclude that there is an $(N-n)$-dimensional convex subset $C$ of $V$ such that for $\left(\alpha_{1}, \ldots, \alpha_{N-n}\right) \in C$ the polytopes $P\left(\alpha_{1}, \ldots, \alpha_{N-n}\right)$ belong to $D_{2}$. It follows that any neighborhood of $[B]$ in $\mathcal{D}_{X}$ contains a convex subset of dimension $N-n$.

As mentioned, Theorem 3 implies that for a polyhedral Minkowski space $X$, the set $\mathcal{D}_{X}$ has only finitely many extreme points. One may conjecture that polyhedral Minkowski spaces are characterized by this fact, but we have only been able to prove this in the two-dimensional case. In higher dimensions, we can show that in Minkowski spaces with a strictly convex norm, the set $\mathcal{D}_{X}$ has infinitely many extreme points. This requires to deal with two tasks: to construct extreme complete sets of given diameter, and for different ones to show that they are not equivalent by translation. First we consider strictly convex norms.

Lemma 7 Let $X$ be a Minkowski space with a strictly convex norm. Let $\emptyset \neq S \subset X$ be a set of vectors of norm 2 with $\operatorname{diam} S \leq 2$, and let $C$ be a completion of minimal volume of $S_{o}:=S \cup\{o\}$. Then $[C]$ is an extreme element of $\mathcal{D}_{X}$.

Proof Since the set $\gamma\left(S_{o}\right)$ of completions of $S_{o}$ is compact, it is clear that a completion $C \in \gamma\left(S_{o}\right)$ of minimal volume exists. We assert that $C$ is extreme, in the set of complete sets of diameter 2 (notice that $\operatorname{diam} C=\operatorname{diam} S_{o}=2$ ). For the proof, let $K, M \subset X$ be two complete sets of diameter 2 such that

$$
C=(1-\lambda) K+\lambda M
$$

for some $\lambda \in(0,1)$. For $x \in S_{o}$, there exist points $x_{K} \in K$ and $x_{M} \in M$ such that $x=$ $(1-\lambda) x_{K}+\lambda x_{M}$. We assume, without loss of generality, that $o_{K}=o_{M}=o$, since this can be achieved by applying suitable translations to $K$ and $M$ without destroying the relation (10). From $\operatorname{diam} K=\operatorname{diam} M=2$ it then follows that $\left\|x_{K}\right\| \leq 2$ and $\left\|x_{M}\right\| \leq 2$. For $x \in S$ we obtain

$$
2=\|x\|=\left\|(1-\lambda) x_{K}+\lambda x_{M}\right\| \leq(1-\lambda)\left\|x_{K}\right\|+\lambda\left\|x_{M}\right\| \leq 2 .
$$

Since equality holds and the norm is strictly convex, we deduce that $x_{K}=x_{M}=x$. Thus, $S_{o} \subset K$ and $S_{o} \subset M$, therefore $K$ and $M$ are completions of $S_{o}$. Since $C$ is a minimum volume completion of $S_{o}$, the volumes of $K, M, C$ satisfy $V(K) \geq V(C)$, $V(M) \geq V(C)$. From (10) and the Brunn-Minkowski theorem it follows that

$$
V(C)^{1 / n} \geq(1-\lambda) V(K)^{1 / n}+\lambda V(M)^{1 / n} \geq V(C)^{1 / n} .
$$

Since equality holds here, the bodies $K$ and $M$ are homothetic and hence, having the same diameter, are translates of each other. This entails that $[C]$ is an extreme element of $\mathcal{D}_{X}$. 
The proof of Lemma 7 leads us to the following side-remark on the so-called Meissner bodies in Euclidean three-space $\ell_{2}^{3}$. To obtain them, one starts with a regular tetrahedron $T$ of edge length $d$, forms the intersection of the balls of radius $d$ with centers at the vertices of $T$, and then rounds off one edge from each opposite pair of curved edges to obtain a body of constant width $d$. These bodies were introduced by Meissner [11], their construction is described in Bonnesen-Fenchel [1, p. 136]; a photograph of a plaster model can be found in Fischer [4, p. 98]. They are famous due to the fact that they have been conjectured (Bonnesen-Fenchel, loc. cit.) to be the bodies of constant width $d$ of minimal volume; this is still undecided. The problem, its history and some recent developments are neatly presented in the article by Kawohl and Weber [8]. The conjecture would be disproved if it could be shown that a Meissner body does not define an extreme class in $\mathcal{D}_{\ell_{2}^{3}}$; however, this approach fails.

Proposition 1 The translation class of a Meissner body is extreme in $\mathcal{D}_{\ell_{2}^{3}}$.

Proof We make use of the following easily proved facts. (i) If $C$ is a subset of diameter $d$ in a normed space with unit ball $B$ and if there is a subset $C^{\prime} \subset C$ satisfying

$$
\operatorname{diam} \bigcap_{t \in C^{\prime}}(d B+t)=d,
$$

then $D:=\bigcap_{t \in C^{\prime}}(d B+t)$ is the only completion of $C$; (ii) If $A=\operatorname{conv}\left\{a_{1}, a_{2}\right\}$, $B=\operatorname{conv}\left\{b_{1}, b_{2}\right\}, C=\operatorname{conv}\left\{c_{1}, c_{2}\right\}$ are segments in a strictly convex normed space satisfying $\left\|a_{1}-a_{2}\right\| \geq\left\|b_{1}-b_{2}\right\|,\left\|c_{1}-c_{2}\right\|$ and $a_{i}=\lambda b_{i}+(1-\lambda) c_{i}, i=1,2$, for some $\lambda \in(0,1)$, then $B$ and $C$ are translates of $A$.

There are two types of Meissner body, according to which three curved edges have been rounded: three that have a common vertex or three that form a triangle. First we consider the case of a common vertex. Let $B$ be the unit ball of $\ell_{2}^{3}$. Without loss of generality, we take the plane $H:=\left\{\left(x_{1}, x_{2}, x_{3}\right) \in \mathbb{R}^{3}: x_{3}=-2 \sqrt{2 / 3}\right\}$ and let $\left\{a_{1}, a_{2}, a_{3}\right\} \in H \cap \partial 2 B$ be three points forming with $o$ an equilateral set of diameter 2 . If we consider the sets

$$
M_{0}:=\left(2 B+a_{1}\right) \cap\left(2 B+a_{2}\right) \cap\left(2 B+a_{3}\right) \cap \partial 2 B
$$

and

$$
M_{1}:=\bigcap_{t \in M_{0}}(2 B+t),
$$

then $M_{1}$ is a Meissner body with the rounded edges having $o$ as common vertex. Moreover, $M_{1}$ is the only completion of $M_{0}$ and hence, by Lemma 7, the translation class $\left[M_{1}\right]$ is an extreme element of $\mathcal{D}_{\ell_{2}^{3}}$.

In order to deal with the second type of Meissner body, consider the halfspace $H^{+}:=\left\{\left(x_{1}, x_{2}, x_{3}\right) \in \mathbb{R}^{3}: x_{3} \geq-2 \sqrt{2 / 3}\right\}$ and define the new sets

$$
M_{0}^{\prime}:=\left(2 B+a_{1}\right) \cap\left(2 B+a_{2}\right) \cap\left(2 B+a_{3}\right) \cap H^{+}
$$

and

$$
M_{1}^{\prime}:=\bigcap_{t \in M_{0}^{\prime}}(2 B+t) .
$$


Then $M_{1}^{\prime}$ is the only completion of $M_{0}^{\prime}$ and is a Meissner body of the second type, with the rounded edges forming a triangle. We state that $M_{1}^{\prime}$ is extreme, up to translations, in the family of complete sets of diameter 2 . In fact, suppose that $M_{1}^{\prime}$ is a convex combination of complete sets $A$ and $B$ of diameter 2 . Then these two sets contain translates of $M_{0}^{\prime}$ (as follows from fact (ii) above), which implies that $\left[M_{1}^{\prime}\right]=$ $[A]=[B]$.

After this digression, we return to the extreme points of $\mathcal{D}_{X}$ in general.

Theorem 4 Let $X$ be a Minkowski space with a strictly convex norm. Then the set $\mathcal{D}_{X}$ has continuum many extreme points.

Proof In the following proof, the notions of unit normal vector and spherical image refer again to an auxiliary Euclidean metric on $X$.

Let $H$ be a two-dimensional plane through $o$ and let $J:=H \cap \partial 2 B$, where $B$ is the unit ball of $X$. We choose a point $x_{1} \in J$ and let $y_{1}$ be one of the two points in $J \cap\left(J+x_{1}\right)$. Then $\left\|x_{1}-y_{1}\right\|=2$ (this is the known argument to construct an equilateral triangle in a Minkowski plane; see Thompson [15, Theorem 4.1.1]). Let $z \neq x_{1}, y_{1}$ be a point of the arc of $J$ between $x_{1}$ and $y_{1}$. Then $\left\|z-x_{1}\right\|<2$ and $\left\|z-y_{1}\right\|<2$. Let $x_{2}$ be a point of the open arc of $J$ between $x_{1}$ and $z$, and determine $y_{2} \in J$ such that $\left\|y_{2}-x_{2}\right\|=2$ and $y_{1}$ belongs to the open arc of $J$ between $z$ and $y_{2}$. We can choose $x_{2}$ so close to $x_{1}$ that still $\left\|z-y_{2}\right\|<2$; of course, $\left\|y_{2}-x_{1}\right\|>2$.

There is a neighborhood $N$ of $z$ in $X$ such that $\left\|w-x_{i}\right\|<2$ and $\left\|w-y_{i}\right\|<2$ for all $w \in N$ and for $i=1,2$. Now we choose $n$ points $w_{1}, \ldots, w_{n} \in N \cap \partial 2 B$ such that there are outer unit normal vectors $u_{i}$ of $2 B$ at $w_{i}, i=1, \ldots, n$, which are linearly independent. That this is possible follows from the fact that $2 B$ is strictly convex. (If $K$ is a strictly convex body, then the spherical image of a relatively open subset of $\partial K$ is a relatively open subset of the unit sphere.)

We define $S_{i}:=\left\{o, x_{i}, y_{i}, w_{1}, \ldots, w_{n}\right\}$ for $i=1,2$, then $\operatorname{diam} S_{i}=2$. Let $C_{i}$ be a minimal volume completion of $S_{i}$. By Lemma $7,\left[C_{i}\right]$ is an extreme point of $\mathcal{D}_{X}$. Suppose that $C_{1}$ and $C_{2}$ were translates of each other. For $j \in\{1, \ldots, n\}$ we have $C_{i} \subset 2 B+w_{j}$, hence $o \in \partial C_{i}$, and $-u_{j}$ is an outer normal vector to $C_{i}$ at $o$. The point $o$ is the only point of $C_{i}$ at which the normal cone contains the linearly independent vectors $-u_{1}, \ldots,-u_{n}$. It follows that $C_{1}$ and $C_{2}$, which are translates, must be identical. But then $x_{1}, y_{2} \in C_{i}$, which is a contradiction, since $\left\|x_{1}-y_{2}\right\|>2$. This proves that $C_{1}$ and $C_{2}$ are not translates of each other. By varying the points $x_{1}$ and $x_{2}$ in suitable neighborhoods, we obtain continuum many different extreme points of $\mathcal{D}_{X}$.

Theorem 5 In a two-dimensional Minkowski space, there are infinitely many extreme translation classes of bodies of given constant width if and only if the unit ball of the space is not a polygon.

Proof Recall that in a two-dimensional Minkowski space, the notions of body of constant width and of diametrically complete set are equivalent. It follows from Theorem 3 that in a polyhedral Minkowski plane there are only finitely many extreme 
translation classes of bodies of given constant width. To prove the converse, we may restrict ourselves to convex bodies of constant width one. Similarly as in the previous proof, we choose a point $x \in \partial B$ (which we call the 'starting point') and let $y$ be a point in $\partial B \cap(\partial B+x)$. Then $\|x\|=\|y\|=\|x-y\|=1$. We remark that $\partial B \cap(\partial B+x)$ contains precisely two points except if $\partial B$ contains a segment parallel to $x$ and of length larger than one. Let

$$
C:=B \cap(B+x) \cap(B+y) .
$$

Of any two parallel supporting lines of $C$, at least one passes through one of the points $o, x, y$, and it follows that the lines are distance one apart. Thus, $C$ is a body of constant width one. The bodies obtained in this way, and their translates, are called Minkowski Reuleaux triangles (although they can have more than three vertices).

Suppose that

$$
C=(1-\lambda) K+\lambda M
$$

for some $K, M \in \mathcal{K}^{2}$ of constant width one and for some $\lambda \in(0,1)$. As in the proof of Lemma 7, there are points $o_{K}, x_{K}, y_{K} \in K$ and $o_{M}, x_{M}, y_{M} \in M$ such that $o=$ $(1-\lambda) o_{K}+\lambda o_{M}, x=(1-\lambda) x_{K}+\lambda x_{M}, y=(1-\lambda) y_{K}+\lambda y_{M}$. Moreover, since $y \in \partial C$, we have $y_{K} \in \partial K, y_{M} \in \partial M$, and the outer unit normal vectors (with respect to some scalar product) of $C$ at $y$, of $K$ at $y_{K}$ and of $M$ at $y_{M}$ are the same. After applying suitable translations we may assume that $o_{K}=o_{M}=o$; then $\left\|x_{K}\right\| \leq 1$, $\left\|y_{K}\right\| \leq 1$. In

$$
1=\|x\|=\left\|(1-\lambda) x_{K}+\lambda x_{M}\right\| \leq(1-\lambda)\left\|x_{K}\right\|+\lambda\left\|x_{M}\right\| \leq 1
$$

we have equality. Equality in the second inequality yields $\left\|x_{K}\right\|=\left\|x_{M}\right\|=1$, hence $x_{K}, x_{M} \in \partial B$. Similarly, we obtain $\left\|y_{K}\right\|=\left\|y_{M}\right\|=1$. Equality in the first inequality of (12) implies that the segment with endpoints $x_{K}, x_{M}$ lies in the boundary of $B$. Now we introduce a first additional assumption, namely that $x$ was chosen as an extreme point of $B$; then we can conclude that $x_{K}=x_{M}=x$. From

$$
\begin{aligned}
1 & =\|x-y\|=\left\|x-(1-\lambda) y_{K}-\lambda y_{M}\right\|=\left\|(1-\lambda)\left(x-y_{K}\right)+\lambda\left(x-y_{M}\right)\right\| \\
& \leq(1-\lambda)\left\|x_{K}-y_{K}\right\|+\lambda\left\|x_{M}-y_{M}\right\| \leq 1
\end{aligned}
$$

we obtain $\left\|x-y_{K}\right\|=1=\left\|x-y_{M}\right\|$. Since also $\left\|y_{K}\right\|=\left\|y_{M}\right\|=1$, we deduce that $y_{K}, y_{M} \in \partial B \cap(\partial B+x)$. As a second assumption, we assume now that $\partial B$ does not contain a segment parallel to $x$ and of length larger than one. Then $\partial B \cap(\partial B+x)$ consists of precisely two points, one of which is $y$. Since the outer unit normal vectors of $C$ at $y$, of $K$ at $y_{K}$ and of $M$ at $y_{M}$ are the same (and $o, x \in K, M$ ), we conclude that $y_{K}=y_{M}=y$. Thus, $o, x, y \in K$, and since $K$ is of constant width one, we must have $K \subset B \cap(B+x) \cap(B+y)=C$. In fact, equality must hold here, since any proper closed convex subset of $B \cap(B+x) \cap(B+y)$ is not of constant width one. Similarly, we have $M=C$. Thus, $C$ is extreme.

Now we assume that the unit ball $B$ of $X$ is not a polygon; then it has infinitely many extreme points. We say that an extreme point $x$ is suitable if $\partial B$ does not contain a segment of length at least one that is parallel to $x$. Clearly, at most finitely many of the extreme points are not suitable. Let $x_{0}$ be an accumulation point of extreme points. There is a point $z \in \partial B \cap \operatorname{int}\left(B+x_{0}\right)$ such the $\operatorname{arc}$ of $\partial B$ between $x_{0}$ 
and $z$ contains infinitely many extreme points. We choose a cyclic order on $\partial B$, and we take two different suitable extreme points $x_{1}$ and $x_{2}$ from the open arc between $x_{0}$ and $z$ such that $x_{0}, x_{1}, x_{2}, z$ follow each other in cyclic order. We choose $y_{1}$ as the first point in $\partial B \cap\left(\partial B+x_{1}\right)$ after $z$ in cyclic order, and $y_{2}$ is defined similarly, for the starting point $x_{2}$. Let $C_{i}$ be the completion of $\left\{o, x_{i}, y_{i}\right\}, i=1,2$, as constructed above, with starting point $x_{i}$. As shown, $C_{1}$ and $C_{2}$ are extreme bodies of constant width one. Suppose that $C_{2}$ is a translate of $C_{1}$, say $C_{2}+t=C_{1}$ with $t \in \mathbb{R}^{2}$. Clearly, $t \neq o$. Since $x_{2}+t \in C_{2}+t=C_{1} \subset B$ and $x_{2}-t \in C_{1}-t=C_{2} \subset B$, the point $x_{2}$ is not an extreme point of $B$, which is a contradiction. Thus, $C_{1}$ and $C_{2}$ are not translates of each other. It follows that there are infinitely many translation classes of extreme bodies of constant width one.

Example 3 The following example shows that Minkowski Reuleaux triangles are not always extreme. Let the unit ball $B$ of $X$ be an affinely regular hexagon, with vertices $v_{1}, v_{2}, v_{3},-v_{1},-v_{2},-v_{3}$, in cyclic order. Performing the construction of the previous proof with the starting point $x_{1}=v_{1}$, we obtain a Minkowski Reuleaux triangle $C_{1}$ which is an ordinary triangle. Starting with $x_{2}=v_{2}$, we get a different Minkowski Reuleaux triangle $C_{2}$, which is also an ordinary triangle. The hexagon $C:=\frac{1}{2}\left(C_{1}+C_{2}\right)$ is the Minkowski Reuleaux triangle which is obtained when we start with the (non-extreme) point $\frac{1}{2}\left(x_{1}+x_{2}\right)$. Thus, $C$ is a non-extreme Minkowski Reuleaux triangle.

Remark Kallay [7] has proved a necessary and sufficient condition for a convex body of constant width in a two-dimensional Minkowski space to be extreme in the set of all convex bodies of the same constant width. We found the proof given above more direct than an application of this criterion. (Note also that in [7, Theorem 2], the condition $\theta \in[0,2 \pi)$ has to be replaced by $\theta \in[0, \pi)$, as shown by the example of the space $\ell_{\infty}^{2}$, and also by the proof given in [7].)

\section{References}

1. Bonnesen, T., Fenchel, W.: Theorie der konvexen Körper. Springer, Berlin (1934). English translation: L. Boron, C. Christenson, B. Smith (eds.) Theory of Convex Bodies. BCS Associates, Moscow, ID (1987)

2. Chakerian, G.D., Groemer, H.: Convex bodies of constant width. In: Gruber, P.M., Wills, J.M. (eds.) Convexity and Its Applications, pp. 49-96. Birkhäuser, Basel (1983)

3. Eggleston, H.G.: Sets of constant width in finite dimensional Banach spaces. Isr. J. Math. 3, 163-172 (1965)

4. Fischer, G.: Mathematische Modelle. Mathematical Models. Vieweg, Braunschweig (1986)

5. Groemer, H.: On complete convex bodies. Geom. Dedic. 20, 319-334 (1986)

6. Heil, E., Martini, H.: Special convex bodies. In: Gruber, P.M., Wills, J.M. (eds.) Handbook of Convex Geometry, pp. 347-385. North-Holland, Amsterdam (1993)

7. Kallay, M.: The extreme bodies in the set of plane convex bodies with a given width function. Isr. J. Math. 22, 203-207 (1975)

8. Kawohl, B., Weber, C.: Meissner's mysterious bodies. Math. Intell. 33, 94-101 (2011)

9. Martini, H., Swanepoel, K.J.: The geometry of Minkowski spaces-a survey. Part II. Expo. Math. 22, 93-144 (2004)

10. McMullen, P.: Representations of polytopes and polyhedral sets. Geom. Dedic. 2, 83-99 (1973) 
11. Meissner, E., Schilling, F.: Drei Gipsmodelle von Flächen konstanter Breite. Z. Math. Phys. 60, 92-94 (1912)

12. Moreno, J.P., Schneider, R.: Local Lipschitz continuity of the diametric completion mapping. Houst. J. Math., in press

13. Moreno, J.P., Schneider, R.: Diametrically complete sets in Minkowski spaces. Isr. J. Math. (2012). doi:10.1007/s11856-012-0003-6

14. Schneider, R.: Convex Bodies: The Brunn-Minkowski Theory. Cambridge University Press, Cambridge (1993)

15. Thompson, A.C.: Minkowski Geometry. Cambridge University Press, Cambridge (1996) 